\title{
Nuevas tendencias en la didáctica de las lenguas extranjeras aplicadas al entorno profesional: la grabación audiovisual con enfoque interdisciplinar
}

\author{
Ana Fe Gil Serra \\ SusANA Nicolás Román \\ Universidad de Almería
}

Recibido: 16 marzo 2014 / Aceptado: 14 noviembre 2014

ISSN: $1697-7467$

\begin{abstract}
RESUMEN: La didáctica de las lenguas extranjeras aplicadas al ámbito profesional ha experimentado una renovación metodológica sustancial en los últimos años. El aprendizaje autónomo así como el desarrollo de las competencias comunicativa e intercultural se perfilan como propuestas eficaces para la formación del alumno universitario. En este artículo presentamos una experiencia docente interdisciplinar donde la grabación de de un vídeo promocional del sector agroalimentario almeriense se convierte en elemento motivador del aprendizaje de lenguas. Esta actividad pone de manifiesto las ventajas que supone no sólo el aprendizaje autónomo y colaborativo sino la familiarización del estudiante con la industria agroalimentaria almeriense y sus esfuerzos por desarrollar una agricultura sostenible. La evaluación del alumnado y de los docentes confirma la consecución de los objetivos iniciales.

Palabras clave: didáctica de las lenguas extranjeras, grabación audiovisual, aprendizaje autónomo, enfoque interdisciplinar, trabajo en equipo, competencia intercultural.
\end{abstract}

New Trends in Foreign Language Didactics Applied to Professional Environments: Audiovisual Recording with Interdisciplinary Approach

\begin{abstract}
The didactics of foreign languages applied to professional environments has experimented a decisive methodological renovation in the last years. Autonomous learning and the development of communicative and intercultural competences are presented as effective proposals for the adequate training of university students. This article presents the experience of a teaching-learning interdisciplinary activity using the audiovisual recording of a promotional video about the agriculture sector in Almeria as a motivating resource in the language learning process. This activity also states the advantages of autonomous and collaborative learning and the familiarization of the student with the agriculture industry in Almeria and its efforts to develop sustainable agriculture. Students and professors assessment confirm the achievement of the initial objectives.
\end{abstract}

Key words: Foreign languages didactics, audiovisual recording, autonomous learning, teamwork, interdisciplinary approach, intercultural competence. 


\section{INTRODUCCIÓN}

El actual escenario de educación plurilingüe exige nuevos modelos educativos en un intento de satisfacer las necesidades de los alumnos en materia de lenguas extranjeras. El impulso del enfoque comunicativo unido a la demanda de las lenguas con fines específicos persigue una formación integral en idioma no sólo centrada en habilidades lingüísticas sino en la capacitación del alumno para sus necesidades profesionales y académicas.

La creación de la Unión Europea, el desarrollo de una sociedad globalizada y el hecho de que las empresas requieran de sus empleados una mayor flexibilidad y movilidad, son algunos de los factores que han convertido la competencia comunicativa en lenguas extranjeras en una de las más valoradas por las grandes empresas internacionales (Zhao, 2003) y ha propiciado el interés por otros idiomas aplicados al sector empresarial hasta ahora minoritarios, como el alemán. El desarrollo de metodologías innovadoras en conjunción con la creación de recursos audiovisuales inicia un nuevo camino en la adquisición de las competencias comunicativa e intercultural del estudiante universitario.

La formación universitaria europea se sustenta en teorías de aprendizaje y principios metodológicos propuestos hace más de una década por el Consejo de Europa en documentos y herramientas metodológicas como el Marco Común Europeo de Referencia para las lenguas: aprendizaje, enseñanza, evaluación (2001) y el Portfolio Europeo de las Lenguas (2000). Las actuales guías docentes reflejan el esfuerzo del profesorado universitario por desarrollar una metodología sustentada en el constructivismo y que, por tanto, potencia la participación activa y autónoma del estudiante en el desarrollo y evaluación de un proceso de aprendizaje que le permita la adquisición de habilidades propias de su futura actividad profesional mediante tareas que favorecen el aprendizaje colaborativo y fuera del aula.

La experiencia docente que analizamos a continuación sobre dos vídeos promocionales del sector agroalimentario almeriense -actualmente disponible en www.hortyfruta.es y www. ceia3.es - sigue las pautas de los estudios cualitativos de acción e investigación, cuyo objetivo es el desarrollo y posterior evaluación de nuevas actividades didácticas que respondan más adecuadamente a las necesidades futuras del estudiante en un marco empresarial, donde las tecnologías de la información y comunicación resultan imprescindibles.

\section{Marco teórico}

\subsection{Adquisición de competencias profesionales: autonomía del aprendizaje y la competencia existencial}

El desarrollo de la autonomía ha supuesto la superación del enfoque lingüístico -que ha dominado durante las últimas décadas- por una nueva dirección en la que la competencia existencial del alumno se considera el objetivo prioritario del docente, que define su actividad como la enseñanza al alumno a ser usuario competente en ese idioma extranjero y a ser actor intercultural (Kohonen, 2012). Este nuevo enfoque convierte en objetivos de aprendizaje la adquisición y/o perfeccionamiento de otras competencias transferibles y profesionales como la capacidad de decisión, la actitud crítica, la autoevaluación, el trabajo en equipo, la competencia intercultural y la actitud responsable en las tareas (Funk, 2007: 177; Funk 
y Kuhn, 2010: 320). Así pues, podemos definir la competencia profesional del estudiante de lenguas extranjeras académicas como la capacidad de hacerse comprender y actuar en el contexto cotidiano y ámbito profesional según las correspondientes situaciones comunicativas para alcanzar determinados objetivos. Según plantean McHann y Frost, el estudiante debe superar el denominado "knowing-gap", la diferencia real entre el conocimiento teórico y la aplicación que el alumno puede ejecutar en la práctica:

The knowing-gap is the critical difference between what a student knows and what he or she can actually do. In order to close the gap, students must develop a disciplined habit of learning so that a feedback loop is established so that, long after graduation, students not only know, but also can actually do (en Hodge, 2014: 2).

El desarrollo de la competencia existencial aludido por Kohonen se refleja en una metodología de enseñanza en la que el trabajo autónomo del estudiante sólo será efectivo si va acompañado de un proceso de autoevaluación de estos resultados. Asimismo, y dado que los actuales estudios de Grado plasman la relevancia del trabajo fuera del aula por parte del estudiante, una de las tareas prioritarias del docente de lenguas extranjeras es ofrecer tareas motivadoras que promuevan el trabajo individual y colaborativo (Herrmann y Siebold, 2012), así como dotar al estudiante de herramientas que faciliten la aplicación de estrategias de aprendizaje y su labor autoevaluadora de los resultados obtenidos.

Para desarrollar la autonomía del aprendizaje es necesario que el estudiante desarrolle una determinada relación psicológica con el proceso y contenido de aprendizaje (Esteve, 2012). No es suficiente que el alumno reconozca que es responsable de su propio aprendizaje y del control del proceso fijándose objetivos y eligiendo actividades y materiales de aprendizaje (Cots y Nussbaum, 2002; van Lier, 2004). Los alumnos deben evaluar sus resultados de aprendizaje, identificando los puntos débiles y fuertes para poder focalizar la siguiente fase de aprendizaje (Little, 2008).

El Portfolio Europeo de las Lenguas se ha convertido en una de las herramientas más eficaces para el desarrollo de la autonomía y autoevaluación en el aprendizaje de idiomas extranjeros. Su estructura se conforma en tres bloques:

- Pasaporte Lingüístico: actualizado regularmente por el titular, refleja el nivel de competencia comunicativa del alumno en cada una de las destrezas, incluyendo además experiencias de aprendizaje formal y no formal.

- Biografía Lingüística: describe el proceso de aprendizaje del alumno facilitándole la planificación y evaluación de su progreso. Las tablas de autoevaluación con sus correspondientes descriptores ofrecen la posibilidad de autoevaluar el nivel de competencia del estudiante y reflexionar sobre su proceso de aprendizaje. Asimismo, contiene información sobre diplomas obtenidos, cursos realizados y experiencias de aprendizaje no formal.

- Dossier: incluye certificados y diplomas que acreditan el nivel de competencia comunicativa del usuario en lengua(s) extranjera(s) así como las actividades de aprendizaje más significativas y que demuestran el nivel de competencia adquirido. 
El Portfolio Europeo de las Lenguas en su versión electrónica (e-PEL) permite a los estudiantes planificar y adecuar su aprendizaje a unas premisas de calidad que facilitan la movilidad académica y profesional. Las principales ventajas de esta herramienta, impulsada por el Consejo de Europa, destacan la transparencia al usar el Marco Común Europeo de Referencia, la transferibilidad de sus resultados fuera de la educación formal y la reflexión del usuario sobre su competencia en lengua(s) extranjera(s), que favorece el aprendizaje autónomo (Canga Alonso, 2011; Gil Serra y Nicolás Román, 2012a: 921).

Asimismo, al incluir en la Biografía la competencia intercultural el e-PEL pone de manifiesto que una de las tareas prioritarias en este ámbito académico es "practicar durante el aprendizaje no sólo el funcionamiento de la lengua extranjera, sino preparar también a los estudiantes para estos contactos internacionales y - de forma transversal- conseguir el objetivo de aceptar las diferencias entre los seres humanos, descubrir una nueva cultura con ayuda del idioma y de aprender a ver la suya con otros ojos" (Neuner, 2007: 232). Cuando los estudiantes comienzan a crear un sentido de comunidad y perciben la diversidad cultural, aprenden a aceptar los comportamientos y actitudes del otro como elemento del proceso de adquisición de habilidades interpersonales (Hodge, 2014). El desarrollo de la competencia intercultural, necesario en el aprendizaje de una lengua extranjera, resulta imprescindible en el ámbito empresarial internacional (Vigier, 2011). Como señalaban directivos de las empresas Siemens y Porsche en las jornadas organizadas por el Goethe Institut de Chicago en 2003 dirigidas a docentes universitarios,

we aren't looking for American students who speak excellent German; we need employees who have studied intercultural communication and can handle the specific contexts of the German-speaking world. The language is secondary; educate your students about the significant cultural differences! (Finger, 2005:78).

\subsection{Didáctica de las lenguas extranjeras y su aplicación en el ámbito empresarial}

La adquisición de otras competencias profesionales se manifiesta en una didáctica en la que, además de la utilización de material propio de las actividades futuras a desarrollar por los estudiantes (Keller, 2006; Schlickau, 2007) priman los "escenarios profesionales", que ofrecen la posibilidad de trabajar sobre situaciones y problemas reales de este entorno profesional (Bryant, 2007; Babanoury, 2005) y de desarrollar competencias como la capacidad analítica, la solución de problemas, el trabajo en equipo y aumentar la motivación del alumnado (Wickum y Kiefer, 2007). Esta metodología está ampliamente difundida en las escuelas de negocios y permite la integración de herramientas didácticas habituales en la enseñanza de lenguas extranjeras como el juego de rol y el análisis textual, que favorecen la adquisición y práctica del léxico especializado (Gonglewski, 2010). Universidades de reconocido prestigio como Harvard o Berkeley, incorporan modelos experimentales a través de situaciones de aprendizaje de experiencia real. Estos nuevos enfoques apuestan por una "aplicabilidad social" en metodologías docentes más "reflexivas, orientadas a la acción y de naturaleza -adquirir-interpretar-aplicar" (Hodge, 2014: 9).

Actualmente, las facultades que ofertan en sus Títulos de Grado y Máster el aprendizaje de las lenguas extranjeras aplicadas al ámbito empresarial incentivan la colaboración entre las distintas áreas de conocimiento presentes en dichos títulos (Gonglewski y Helm, 2013; 
Babanoury, 2005), así como el acercamiento a la realidad del entorno local e internacional (Doyle, 2012) mediante la cooperación con empresas, proyectos de desarrollo y visitas de expertos internacionales (Bryant, 2007; Grosse, 2005). Son numerosas también las facultades que integran conceptos como ecología y sostenibilidad en su oferta académica (Gonglewski y Helm, 2013), adaptándose a las necesidades del mundo empresarial y expectativas del alumnado. Un reciente estudio realizado en varias universidades norteamericanas señala que el $83 \%$ de los estudiantes desearían que se incluyeran "green contents" en los cursos de gestión y más del $76 \%$ en cursos de marketing y comercio exterior (Silverblatt y Bates, 2012).

La didáctica de las grabaciones audiovisuales como recurso para desarrollar la comprensión oral en L2 ha sido desarrollada por numerosos estudios (Brown y Yule, 1983; Ur, 1984, 1997; Rost, 2002; Cassany, Luna y Sanz, 1993; Nunan y Miller, 1995; Flowerdew y Miller, 2005). En todos ellos se recogen actividades basadas en materiales de vídeo destacando además los principales beneficios de las grabaciones audiovisuales. Según Calzadilla, la elaboración de vídeos permite desarrollar el trabajo colaborativo así como la autonomía del estudiante: "cada participante asume su propio ritmo y potencialidades, impregnando la actividad de autonomía, pero cada uno comprende la necesidad de aportar lo mejor de sí al grupo para lograr un resultado sinérgico, al que ninguno accedería por sus propios medios" (2004: 4).

Por otro lado, también encontramos aportaciones más específicas destinadas a la producción de videos en el aula de lenguas extranjeras con la finalidad de ofrecer propuestas a la didáctica de la comprensión oral (Lonergan, 1984; Tomalin, 1986; Arcario, 1991; Stoller, 1991; Bustos, 1997; Corpas, 2000; Stempleski y Tomalin, 2001). La experiencia docente que desarrollamos a continuación se apoya en esta línea utilizando la creación de vídeos por parte de los estudiantes como recurso de aula para mejorar la expresión oral en lengua extranjera.

\section{EXPERIENCIA DOCENTE: GRABACIÓN DE VIDEO PROMOCIONAL}

La actividad que presentamos a continuación se realizó durante el curso académico 2012/13 como resultado de un proyecto de innovación docente impulsado por el Campus de Excelencia Agroalimentario ceia3 entre docentes de Filología Inglesa y Alemana y el Departamento de Producción Vegetal de la Universidad de Almería. Se les propuso a los estudiantes de las asignaturas "Wirtschaftsdeutsch I" y "English Business" del Máster en Gestión Internacional e Idiomas Extranjeros elaborar dos vídeos promocionales -uno en inglés y otro en alemán- sobre el sector agroalimentario almeriense. Ambos vídeos serían posteriormente distribuidos en campañas publicitarias sobre el sector agroalimentario en el ámbito nacional e internacional.

\subsection{Participantes}

Dado el alto grado de interés de los alumnos por participar en dicho proyecto, se llevó a cabo un proceso de selección en función de los resultados presentados en la autoevaluación realizada utilizando el e-PEL. En dicho proceso, se valoraron, además del nivel de competencia lingüística, las experiencias de aprendizaje no formal, la competencia intercultural, la capacidad de trabajo en equipo y la responsabilidad en la realización de tareas. Se crearon un grupo de lengua inglesa y otro de lengua alemana constituido cada uno de ellos 
por cuatro estudiantes. Cada grupo contó con un alumno nativo como apoyo lingüístico así como dos alumnos de doctorado del Departamento de Producción Vegetal, que asesoraron a ambos grupos en cuestiones relativas a la producción agroalimentaria bajo invernadero.

\subsection{Planificación de objetivos}

Con objeto de determinar los objetivos de aprendizaje de esta actividad, se establecieron previamente una serie de líneas de actuación basadas en el método de estudio investigaciónacción participativa, que identifica al participante como parte central del proceso y propone una exploración previa, una intervención didáctica por parte del docente y una reflexión final que incluye a todos los miembros integrantes en el estudio y donde se plantean limitaciones y proyectos de futuro (Mackey y Gass, 2012). Durante esta fase previa, se delimitaron las principales competencias profesionales requeridas por los alumnos y se fijaron los siguientes objetivos de aprendizaje:

1. Mejorar las competencias intercultural y comunicativa en lengua extranjera (inglés y alemán) aplicada al ámbito empresarial.

2. Desarrollar competencias profesionales relacionadas con la promoción internacional de productos agroalimentarios.

3. Aplicar adecuadamente conceptos propios de la producción agroalimentaria bajo invernadero como sostenibilidad, control biológico, producción integrada y ecológica en la formación de nuestros estudiantes.

4. Identificar las principales fortalezas y debilidades del trabajo en equipo realizado.

La grabación de ambos vídeos se llevó a cabo en el Estudio Polimedia del Centro de Producción de Contenidos Digitales de la Universidad de Almería. El sistema utilizado para la producción audiovisual, Croma Key, consiste en la captación de una imagen (persona u objeto) delante de un fondo liso y uniforme, reemplazado por un fondo alternativo añadido digitalmente utilizando el proceso keying. El Kit de Reflecmedia del que dispone el Centro de Producción de la UAL permite mejorar la calidad de la posproducción. Se utilizaron escenarios reales de la provincia de Almería, así como el propio Centro de Producción de la Universidad para la grabación de escenas relativas a los procesos agroalimentarios, o las condiciones climáticas y geográficas de la región.

El proyecto colaborativo de esta actividad se estructuró en las siguientes fases:

- Briefing sobre el proyecto y los objetivos a alcanzar, en el que participaron la Técnico Audiovisual del Campus de Excelencia Agroalimentario ceia3, de la Gerente de la empresa Hortyfruta y las docentes responsables de la actividad.

- Visita a empresas representativas del sector agroalimentario almeriense.

- Sesión formativa sobre calidad y seguridad alimentaria a cargo del Departamento de Producción Vegetal y visita a dos laboratorios.

- Elaboración de las propuestas por parte de los dos grupos de estudiantes.

- Presentación de las propuestas publicitarias de ambos grupos ante las docentes responsables de la actividad y la Gerente de la empresa Hortyfruta.

- Realización de los dos vídeos promocionales en las instalaciones audiovisuales de la Universidad de Almería con el asesoramiento de las docentes responsables.

- Presentación ante los medios de comunicación de ambas campañas publicitarias. 
La realización de este proyecto, en el que también participaba la empresa Hortyfruta -Organización Interprofesional de frutas y hortalizas de Andalucía- respondía a los principios metodológicos señalados, proponiendo una tarea atractiva al estudiante universitario que le permitía el análisis y debate de distintas propuestas. La elaboración de estos vídeos nos permitía vincular el perfeccionamiento de la competencia comunicativa a la adquisición de la competencia intercultural (visión del consumidor inglés y alemán respecto de los productos españoles) además de adecuar los objetivos de aprendizaje de ambas lenguas extranjeras a las expectativas de los estudiantes, cada vez más interesados en el desarrollo de una industria ecológica y sostenible. Asimismo, y dado que ambas docentes utilizábamos en nuestras asignaturas el e-PEL, los estudiantes podían evaluar en qué medida el desarrollo de dicha actividad les permitía alcanzar las capacidades señaladas en los descriptores incluidos en las tablas de autoevaluación en la sección de "Biografía" (Gil Serra y Nicolás Román, 2012b: 503).

\subsection{Análisis de las grabaciones audiovisuales}

Las grabaciones realizadas por ambos grupos seguían una misma línea argumentativa. Con objeto de rebatir la imagen negativa de nuestros productos -principalmente en el mercado alemán y austriaco- los estudiantes eligieron como motivo central de la campaña a una madre joven acompañada de su hija comparando verduras en un supermercado. Ambos vídeos se inician con los mismos personajes -en el clip de lengua inglesa, se sitúa a los actores en la ciudad de Ilkley y en el clip de lengua alemana, en la ciudad de Munich. Estos dos personajes despiertan en el espectador desde el inicio la confianza en la calidad y frescura de los productos almerienses. Cada una de las preguntas de la niña da paso a una serie de secuencias en las que los estudiantes relatan las características de producción en los invernaderos, la aplicación de controles la calidad, la experiencia de la industria agroalimentaria almeriense durante las últimas décadas y la seguridad que garantiza la trazabilidad de los productos desde el origen hasta el mercado de destino. La calidad y el sabor de los productos almerienses cierran ambos videos publicitarios mediante una escena en la que los personajes de la madre y la hija preparan la comida en la cocina de su casa. Ambas grabaciones finalizan con una escena conjunta de los dos grupos de estudiantes en las playas de Almería.

Las diferencias culturales de ambos mercados fueron valoradas por los dos grupos y se plasman en diferencias significativas tanto en la estructura de los videos resultantes como en las referencias a las características de la producción, el control de calidad, los beneficios de la producción biológica y los efectos medioambientales.

Podemos distinguir tres grandes bloques temáticos en ambas grabaciones. El primer bloque está dedicado a la producción en invernaderos y manipulación de productos. El segundo bloque se centra en las características medioambientales de Andalucía y la provincia de Almería así como en su experiencia en la industria agroalimentaria. El tercer y último bloque hace referencia a las condiciones de transporte entre el mercado de origen y los mercados europeos de destino. Las mayores diferencias entre ambas grabaciones se producen en el primer bloque temático. El video destinado al mercado británico comienza utilizando el escenario del laboratorio, donde se explica el control biológico de plagas y la utilización de abejorros en el proceso de polinización. El video destinado al mercado alemán 
sigue la estructura temporal de la producción agroalimentaria, iniciándose en este caso con la construcción de los semilleros, producción en invernaderos, selección de productos y trazabilidad indicada en el etiquetado, como herramienta del control de calidad. En cada uno de estos escenarios se destaca la aplicación de la normativa europea que asegura la calidad de los productos. A diferencia del vídeo realizado por los estudiantes en lengua inglesa, no se menciona la polinización mediante abejorros, pero sí se destacan el reciclaje de plásticos y vegetales así como la utilización del riego por goteo como medidas medioambientales relevantes de la producción agroalimentaria almeriense.

Tabla 1. Diferencias en la selección de escenarios vídeo lengua inglesa/alemana

\begin{tabular}{|c|c|}
\hline Grabación en lengua inglesa & Grabación en lengua alemana \\
\hline $\begin{array}{l}\text { Escenario 1: Laboratorio de Universidad de } \\
\text { Almería y fondos con escenas de abejorros. } \\
\text { Discurso sobre polinización y control de plagas. }\end{array}$ & $\begin{array}{l}\text { Escenario 1: Fondos de escenas sobre semilleros. } \\
\text { Discurso sobre construcción según normas de } \\
\text { calidad de la EU. }\end{array}$ \\
\hline $\begin{array}{l}\text { Escenario 2: Invernaderos. } \\
\text { Discurso sobre manipulado de productos } \\
\text { siguiendo pautas tradicionales. Implicación de } \\
\text { profesionales en calidad de productos. }\end{array}$ & $\begin{array}{l}\text { Escenario 2: Invernaderos. } \\
\text { Discurso sobre fases de preparación del suelo } \\
\text { antes de la plantación. Los controles de calidad } \\
\text { europeos certifican la calidad de los productos. } \\
\text { Ventajas del riego por goteo. }\end{array}$ \\
\hline \multirow[t]{2}{*}{$\begin{array}{l}\text { Escenario 3: Laboratorio de la Universidad de } \\
\text { Almería. Discurso explicativo sobre la } \\
\text { trazabilidad. }\end{array}$} & $\begin{array}{l}\text { Escenario 3: Fondos de escenas sobre } \\
\text { manipulación de verduras. } \\
\text { Discurso sobre el proceso de cultivo y selección. } \\
\text { Producción y control biológico según normativa } \\
\text { nacional y europea. Descripción del cuaderno de } \\
\text { campo y trazabilidad. }\end{array}$ \\
\hline & $\begin{array}{l}\text { Escenario 4: Laboratorio de la Universidad de } \\
\text { Almería. Discurso sobre reciclaje de plásticos y } \\
\text { plantas. }\end{array}$ \\
\hline
\end{tabular}

El bloque temático relativo a la provincia de Almería es muy similar en ambas grabaciones, ya que utilizan los mismos fondos explicativos que aluden a la imagen turística más conocida de la provincia. La única diferencia significativa se centra en el énfasis de las ventajas climatológicas como situación geográfica, horas anuales de luz solar y 50 años de experiencia en la industria agroalimentaria para el video en lengua inglesa mientras que el vídeo en lengua alemana -teniendo en cuenta que Holanda es el mayor competidor de los productos almerienses en el mercado alemán- menciona además que estas características climatológicas permiten que no sea necesaria la utilización de luz artificial ni calefacción en los meses de invierno, lo que proporciona ventajas a nuestros productos. 


\subsection{Análisis de resultados}

El análisis de los resultados de la actividad detallada anteriormente se centra en:

1. Mejorar las competencias intercultural y comunicativa en lengua extranjera (inglés y alemán) aplicada al ámbito empresarial.

El perfeccionamiento de la competencia comunicativa oral en el ámbito profesional constituía uno de los principales objetivos tanto para alumnos como para docentes. La autoevaluación realizada sobre los objetivos alcanzados mediante esta actividad se realizaron utilizando descriptores del nivel $\mathrm{C} 1$ y C2 del e-PEL en el caso de los estudiantes del lengua inglesa. En los resultados se plasma un mayor dominio de capacidades propias de este nivel como: "Soy capaz de conversar con fluidez, corrección y precisión, sobre una amplia gama de temas generales, profesionales o académicos/ Soy capaz de presentar descripciones claras y detalladas sobre temas complejos de mi especialidad, integrando diversos temas, desarrollando determinados puntos y concluyendo de forma apropiada". Es necesario destacar que el nivel de competencia comunicativa de los estudiantes españoles de lengua alemana era inferior a los estudiantes no nativos de lengua inglesa. Pese al esfuerzo realizado se perciben algunas dificultades en pronunciación, dicción y prosodia. No obstante, la repetición y la memorización de pequeños fragmentos permitieron un resultado final que los propios estudiantes calificaron de muy satisfactorio. Este grupo de estudiantes señaló como objetivos alcanzados mediante esta actividad descriptores incluidos en el e-PEL correspondientes al nivel B2 como: "Soy capaz de informar o hacer una descripción clara y detallada sobre una amplia variedad de temas de mi interés o relacionados con mi campo de especialización/ Tengo recursos para resaltar la información que considero más importante". Los estudiantes nativos de lengua inglesa y alemana evaluaron positivamente la labor de apoyo y asesoramiento que habían realizado con los estudiantes españoles y manifestaron que, pese a no poder evaluar su mejora en el nivel de competencia comunicativa en lengua española, esta actividad les había permitido reflexionar sobre su propia lengua y cultura. Asimismo, todos los alumnos valoraron muy positivamente el aprendizaje de la comunicación no verbal en el sector audiovisual, y el desarrollo de habilidades dramáticas.

La realización de estos vídeos promocionales puso de manifiesto la diferencia entre los conceptos de competencia intercultural, definida como la habilidad de interactuar en la propia lengua materna con personas de otro país y cultural, y la competencia comunicativa intercultural, como la habilidad de interactuar adecuadamente en una lengua extranjera (Bryan, 1997). La adquisición y perfeccionamiento de ambas se vio favorecida por la diversidad nacional de los componentes de ambos equipos. Ha de destacarse también el desarrollo de la competencia transcultural entre los estudiantes nativos, ya que esta actividad les permitió reflexionar sobre las características del consumidor de lengua inglesa y alemana, valorando también de forma significativa el conocimiento adquirido sobre el sector agroalimentario almeriense.

2. Desarrollar competencias profesionales relacionadas con la promoción internacional de productos agroalimentarios.

Dado que los objetivos de aprendizaje indicados abarcaban no sólo las competencias lingüísticas e interculturales adquiridas por los estudiantes, para la evaluación 
de los resultados obtenidos se utilizaron además del e-PEL un cuestionario online y la entrevista personal con las profesoras responsables de la actividad. Como se ha señalado previamente, ambas propuestas publicitarias fueron evaluadas previamente por expertos en la materia, en las que se constató que los alumnos habían desarrollado competencias profesionales propias de la promoción de productos nacionales en un entorno internacional. La actividad evidencia que la creación de estos vídeos promocionales producidos por la Unidad de Contenidos Digitales y Multimedia permite al alumno realizar actividades de aprendizaje relacionadas con su futura actividad profesional, familiarizarse con uno de los sectores empresariales más significativos de la provincia de Almería y perfeccionar su competencia comunicativa en lengua extranjera. Asimismo, tanto la entrevista personal como el cuestionario online pusieron de manifiesto que la motivación del alumno en el aprendizaje de la lengua extranjera se vio acrecentada al convertirse en co-autor de un material accesible de forma gratuita en internet. Asimismo, queda demostrado que la creación de equipos interdisciplinares ha permitido a la formación universitaria superar los déficits que originaba una actividad docente e investigadora limitada a la propia área de conocimiento.

3. Aplicar adecuadamente conceptos propios de la producción agroalimentaria bajo invernadero.

Los alumnos y docentes valoraron muy positivamente las sinergias producidas en esta actividad interdisciplinar. La integración de conceptos propios del sector agroalimentario como control biológico, producción integrada, trazabilidad, cuaderno de campo, así como de las investigaciones desarrolladas por el Departamento de Producción Vegetal permitió a los estudiantes reflexionar sobre el esfuerzo producido en este sector durante las últimas décadas. La estimación sobre esta actividad coincide con la conclusión recogida en el informe "Una nueva era de la sostenibilidad: Encuesta 2010 a CEOs Pacto Mundial de las Naciones Unidas-Accenture", según el cual el 93\% de los directivos cree que la sostenibilidad se convertirá en el plazo de 10 años en un factor clave para el éxito de sus empresas (Accenture, 2010). La reflexión individual con la que finalizaba esta actividad les permitió también a los alumnos analizar el impacto de esta actividad en el aprendizaje de la lengua extranjera (inglés/alemán) y su visión personal respecto de la sostenibilidad y la responsabilidad medioambiental. En realidad, esta reflexión representó la tarea inversa a la realizada previamente, ya que supone detenerse en qué y cómo han aprendido frente a la aplicación práctica de lo aprendido.

4. Identificar las principales fortalezas y debilidades del trabajo en equipo realizado.

Todos los participantes en esta actividad valoraron positivamente la oportunidad que les había brindado esta actividad para desarrollar una visión crítica respecto del trabajo en equipo. Sin embargo, mientras que los miembros del equipo en lengua inglesa destacaron como principales fortalezas de su tarea la comunicación y colaboración entre los integrantes, el equipo en lengua alemana reconoció que la insuficiente comunicación y la ausencia de retroalimentación habían sido dos de las mayores limitaciones les habían impedido obtener 
mejores logros. Las competencias más desarrolladas fueron la actitud crítica respecto al trabajo realizado en equipo. Todos los participantes reconocieron la importancia del enfoque autónomo en este proyecto, aunque reconocieron algunas limitaciones. Se produjo una mayor colaboración y comunicación entre los miembros del equipo de lengua inglesa, pues que su grado de responsabilidad e implicación fue mayor que en el equipo de lengua alemana.

\section{Conclusiones}

Los objetivos propuestos inicialmente por el presente proyecto fueron alcanzados y así lo atestiguan tanto el éxito de los vídeos promocionales en sí como la autoevaluación realizada por los alumnos mediante el e-PEL y las conclusiones señaladas en el cuestionario online y la entrevista personal. El acceso a material de contenido agroalimentario -de especial relevancia empresarial en la zona- fue valorado muy positivamente por los estudiantes. La cooperación interdisciplinar entre la Unidad de Contenidos Digitales y las áreas académicas ya mencionadas, atestigua de forma clara el desarrollo efectivo del trabajo en equipo así como el asesoramiento de docentes y expertos. Esta actividad pone de manifiesto las sinergias que producen la colaboración docente interdisciplinar y el fomento del trabajo autónomo y colaborativo del estudiante. Gracias a este enfoque didáctico se ha conseguido adecuar el aprendizaje de las lenguas extranjeras a las expectativas profesionales de los alumnos, interesados en el desarrollo de una agricultura sostenible.

Los vídeos resultantes de esta experiencia docente fueron cedidos con derechos de autor a la empresa colaboradora Hortyfruta que los ha difundido en diversas actividades de promoción de la agricultura almeriense. La Universidad de Almería organizó además una rueda de prensa mediante la cual los medios de comunicación locales se hicieron eco de ambos vídeos. La realización de estos videos promocionales nos permitió a las docentes implicadas en esta actividad desarrollar una metodología mucho más cercana a las necesidades y expectativas del estudiante gracias a la cooperación con otras áreas de conocimiento y empresas del sector agroalimentario. Actualmente, estamos proyectando nuevas actividades que promuevan la adquisición de competencias comunicativas, interculturales y profesionales, requeridas en el actual marco de la Educación Superior.

\section{REFERENCIAS BIBLIOGRÁFICAS}

Accenture. (2010). A New Era of Sustainability. UN Global Compact Accenture CEO Study 2010, accesible en https://www.unglobalcompact.org/docs/news_events/8.1/UNGC_Accenture_CEO_Study_2010.pdf Consultado el 14 marzo de 2015.

Arcario, P. (1991). "Criteria for selecting video materials", en S. Stempleski Y P. Arcario (eds.), Video in second language teaching: using, selecting and producing video for the classroom. NY: TESOL, 109-121.

Babanoury, C. (2005). "Real World Business Language Task in Action", en The Journal of Language for International Business, 16, 2: 13-23.t 
Brown, G y G. Yule (1983). Teaching the Spoken Language: An approach based on the analysis of conversational English. Cambridge: CUP.

Bryam, M. (1997). Teaching and Assessing Intercultural Communicative Competence. Clevedon: Multilingual Matters.

Bryant, M. et al (2006). "Embedding Language Learning in the Business School Curriculum and Mission", en Gobal Business Language, 11: 71-82.

Bustos Gisbert, J. M. (1997). "Aplicaciones del vídeo a la enseñanza de español como lengua extranjera", en Revista Carabela, 42: 93-105.

Calzadilla, M. E. (2004). "Aprendizaje Colaborativo y tecnologías de la información y la comunicación". Revista Iberoamericana de Educación OEI, accesible en http://www.rieoei. org/322Calzadilla. Consultado el 14 de marzo de 2014.

Canga Alonso, A. (2011). "El Portafolio como recurso para la reflexión y la autoevaluación en alumnos con dificultades de aprendizaje", en Porta Linguarum 16: 137-153.

Cassany, D., M. Luna y G Sanz. (1993). Ensenyar llengua. Barcelona: Graó.

Chen, G. (1990). "Intercultural communication competence: some perspectives of research", en The Howard Journal of Communication, 2: 243-261.

Corpas Viñals, J. (2000). "La utilización del vídeo en el aula de E/LE: el componente cultural", en M. A. Martín Zorraquino et al (eds.) Actas XI Congreso Internacional de ASELE. Zaragoza: Universidad de Zaragoza, accesible en http://cvc.cervantes.es/ensenanza/biblioteca_ele/asele/asele_xi.htm. Consultado el_14 de abril de 2015.

Cots Caimon, J.M. y Nussbaum Capdevila, L. (2002). "Visiones de la conciencia lingüística: del currículo a la práctica en el aula" en Pensar lo dicho: la reflexión sobre la lengua y la comunicación en el aprendizaje de lenguas, Ed. Milenio, 55-70.

Doyle, M.S. (2012). "Business Language Studies in the United States: On Nomenclature, Context, Theory and Method", en The Modern Language Journal 96: 105-121.

Esteve, O. (2012). "The ELP as a mediating tool for the self-regulation in foreign language university contexts: an ethnographic study", en B. Kühn y M. L. Pérez Cavana (eds). Perspectives from the European Language Portfolio: Learner autonomy and self-assessment, 73-100.

Finger, A., y Kathoefer, G. (2005). "The Quest For Intercultural Competence: Interdisciplinary Collaboration and Curricular Change In Business German", en The Journal of Language for International Business, 16. 2: 78-89.

Flowerdew, J y L. Miller. (2005). Second language listening. NY: CUP

Funk, H. (2007). Berufsbezogener Fremdsprachenunterricht. Fremdsprachenunterricht. Tübingen: Francke, 175-179.

Funk, H., y Kuhn, C. (2010). Berufsorientierter Fremdsprachenunterricht. Handbuch Fremdsprachendidaktik. Selleze-Velber: Kallmayer junto con la editorial Klett. 316-321.

Gil Serra, A.F. y Nicolás Román, S. (2012a). "Aplicación del Portfolio Europeo de las Lenguas en la adquisición de la competencia plurilingüe en el entorno universitario", en La investigación en didáctica de la lengua y la literatura: situación actual y perspectivas de futuro. Ed.: Sociedad Española de Didáctica de la Lengua y la Literatura, 915-922.

Gil Serra, A.F. y Nicolás Román, S. (2012b). "La adquisición de la competencia intercultural en el aprendizaje de una lengua extranjera a través del E-PEL”, en E. Soriano Ayala (ed.), El poder de la comunicación en una sociedad globalizad. Almeria: Univ. de Almeria, 491-505.

Gonglewski, M., y Helm, A. (2013). "LaissezFair: A Case for Greening the Business German Curriculum", en Die Unterrichtspraxis/Teaching German, Vol. 46.2: 200-2014.

Gonglewski, M., y Helm, A. (2010). "An Examination of Business Case Methodology: Pedagogical Synergies from Two Disciplines", en Global Business Language, 15: 17-31. 
González Jiménez, A. (2011). "La adquisición de las competencias comunicativas interculturales, emocionales y sociales a través de un programa de intervención", en E. Soriano Ayala (ed.), El valor de la educación en un mundo globalizado. Madrid: La Muralla, 127-150.

Grosse, C. U. (2005). "Strategic Alliances for Business Languages: From the Classroom To the Community", en The Journal of Language for International Business, 16. 2: 39-48.

Hermann, A., y Siebold, K. (2012). "Projektarbeit im universitären DaF-Unterricht: ein wichtiger Schritt in Richtung Lerner-autonomie", en Info DaF 6: 637-650.

Hodge, L. et al. (2014). "From periphery to core: the increasing relevance of experiential learning in undergraduate business education", en Research in Higher Education Journal 26 (October): 1-17.

Keller, R. (2006). Der Geschäftsbericht. Überzeugende Unternehmenskommunikation durch klare Sprache und gutes Deutsch. Wiesbaden: Gabler.

Kohonen, V. (2012). "Developing the autonomy through ELP-oriented pedagogy: exploring the interplay of shallow and deep structures in mayor change within language education" en B. Kühn y M. L. Pérez Cavana (eds) Perspectives from the European Language Portfolio: Learner autonomy and self-assessment. Routledge, 22-42.

Kühn, B., y Pérez Cavana, M. L (eds.) (2012). Perspectives from the European Language Portfolio: Learner autonomy and self-assessment. London: Routledge.

Little, D. (2008). "Learner autonomy in practice: a challenge for university language teaching", en Autonomes Fremdsprachenlernen in Hochschule und Erwachsenenbildung. Bochum: AKS Verlag, 47-63.

Lonergan, J. (1984). Video in Language Teaching, Cambridge: CUP.

Mackey, A., y Gass, S. (2012). Research Methods in Second Language Acquisition. A Practical Guide. London: Wiley-Blackwell.

Marco Común Europeo de Referencia. http://cvc.cervantes.es/ensenanza/biblioteca_ele/marco/. Consultado el 10 junio 2014.

McHann, J. C. y Frost, L. (2010). "Integrating experiential learning into business courses: using learning journals to create living case studies", en American Journal of Business Education 3(8), accessible en http://www.cluteinstitute.com/ojs/index.php/AJBE/article/view/464/451. Consultado el 14 de mayo de 2014.

Neuner, G. (2007). "Vermittlungsmethoden: "Historischer Überblick" en Handbuch Fremdsprachenunterricht. Tübingen: Francke, 225-234.

Nunan, D y L. Miller (eds.) (1995). New ways in teaching listening. Alexandria (EE.UU.): TESOL

Portfolio Europeo de las Lenguas versión electrónica (e-PEL). http: //www.oapee.es/e-pel/. Consultado el 10 junio 2014.

Rost, M. (2002). Teaching and researching listening. Harlow (UK): Pearson Education.

Salaberri, S. (2007). Competencia comunicativa intercultural. Consejería de Educación de la Junta de Andalucia. http://www.juntadeandalucia.es/educacion/portal/com/bin/Contenidos/IEFP/ Publicaciones/PERSPECTIVA_CEP/1209368660027_05_opinion_compet.pdf . Consultado el 10 de mayo de 2014.

Schlickau, S. (2007). "Zur Kulturspezifik medialer Unternehmensaußenkommunikation", en Fremdsprachen in der Berufsfeld - Foreign Languages in the World of Work. Frankfurt/ Main: Lang, 115-131.

Stempleski, S y B. Tomalin. (2001). Film. Oxford: OUP.

Stoller, F. L. (1991). "Using video in theme-based curricula", en S. Stempleski y P. Arcario (eds.). Video in second language teaching: using, selecting and producing video for the classroom. NY: TESOL, 25-46. 
Tomalin, B. (1986). Video, TV and radio in the English class. London: Macmillan Publishers Ltd. Ur, P. (1997). A Course in Language Teaching: practice and theory. Cambridge: CUP.

Ur, P. (1984). Teaching listening comprehension. Cambridge: CUP. (1994, 12a reimp.).

Van Lier, L. (2004). "La segunda lengua: conocimiento, reflexión e interacción", en Nuevas formas de aprendizaje en lenguas extranjeras. Madrid: Ministerio de Educación y Ciencia, 115-132.

Vigier, M., y Smoller, N. (2011). "Promoting Cultural Awareness In An International Business Program Through Foreign Language Communication", en Global Business Languages 16: 43-58.

Wickum, H., y Kiefer, K.-H. (2007). Zum Einsatz betriebswirtschaftlicher Fallstudien in der Sprachbildung. Wirtschaftsdeutsch vernetzt. Neue Konzepte in Materialien. Munich: IUDICUM, 265-288.

Zhao, J. (2003). "Didaktische und methodische Überlegungen über den Wirtschaftsdeutsch-Unterricht", en Fachsprachenlinguistik, Fachsprachendidaktik und interkulturelle Kommunikation. Frankfurt am Main: Lang, 251-262. 